\title{
Investigation of the effects of vatinoxan on somatic and visceral antinociceptive efficacy of medetomidine in dogs
}

\section{Huuskonen, Vilhelmiina}

2020-04

Huuskonen , V , Restitutti , F , Honkavaara , J M , Raekallio , M R , Männikkö , S , Scheinin , M \& Vainio , O M 2020 , ' Investigation of the effects of vatinoxan on somatic and visceral antinociceptive efficacy of medetomidine in dogs ' , American Journal of Veterinary

Research , vol. 81 , no. 4 , pp. 299-308 . https://doi.org/10.2460/ajvr.81.4.299

http://hdl.handle.net/10138/326341

https://doi.org/10.2460/ajvr.81.4.299

unspecified

publishedVersion

Downloaded from Helda, University of Helsinki institutional repository.

This is an electronic reprint of the original article.

This reprint may differ from the original in pagination and typographic detail.

Please cite the original version. 


\section{Investigation of the effects of vatinoxan on somatic and visceral antinociceptive efficacy of medetomidine in dogs}

\author{
Vilhelmiina Huuskonen DVM \\ Flavia Restitutti DVM, PhD \\ Juhana M. Honkavaara DVM, PhD \\ Marja R. Raekallio DVM, PhD \\ Sofia Männikkö MSc \\ Mika Scheinin MD, PhD \\ Outi M. Vainio DVM, PhD
}

Received July 16, 2019.

Accepted October 15, 2019.

From the School of Veterinary Medicine, University College Dublin, Belfield, Dublin 4, Ireland (Huuskonen); Department of Equine and Small Animal Medicine, Faculty of Veterinary Medicine, University of Helsinki, Fl-00100 Helsinki, Finland (Restitutti, Honkavaara, Raekallio, Vainio); Department of Statistics, 4Pharma Ltd, 20520 Turku, Finland (Männikkö); and Institute of Biomedicine, University of Turku and Unit of Clinical Pharmacology, Turku University Hospital, 20520 Turku, Finland (Scheinin). Dr. Restitutti's present address is the Department of Small Animal Medicine and Surgery, School of Veterinary Medicine, St. George's University, Grenada, West Indies.

Address correspondence to Dr. Huuskonen (vilhelmiina.huuskonen@ucd.ie).

\begin{abstract}
OBJECTIVE
To determine whether concurrent vatinoxan administration affects the antinociceptive efficacy of medetomidine in dogs at doses that provide circulating dexmedetomidine concentrations similar to those produced by medetomidine alone.
\end{abstract}

\section{ANIMALS \\ 8 healthy Beagles.}

\section{PROCEDURES}

Dogs received 3 IV treatments in a randomized crossover-design trial with a 2 -week washout period between experiments (medetomidine [20 $\mu \mathrm{g} /$ $\mathrm{kg}$, medetomidine $[20 \mu \mathrm{g} / \mathrm{kg}]$ and vatinoxan $[400 \mu \mathrm{g} / \mathrm{kg}]$, and medetomidine $[40 \mu \mathrm{g} / \mathrm{kg}]$ and vatinoxan $[800 \mu \mathrm{g} / \mathrm{kg}] ;$ M20, M20V400, and M40V800, respectively). Sedation, visceral and somatic nociception, and plasma drug concentrations were assessed. Somatic and visceral nociception measurements and sedation scores were compared among treatments and over time. Sedation, visceral antinociception, and somatic antinociception effects of M20V400 and M40V800 were analyzed for noninferiority to effects of $\mathrm{M} 20$, and plasma drug concentration data were assessed for equivalence between treatments.

\section{RESULTS}

Plasma dexmedetomidine concentrations after administration of M20 and M40V800 were equivalent. Sedation scores, visceral nociception measurements, and somatic nociception measurements did not differ significantly among treatments within time points. Overall sedative effects of M20V400 and M40V800 and visceral antinociceptive effects of M40V800 were noninferior to those produced by $\mathrm{M} 20$. Somatic antinociception effects of M20V400 at 10 minutes and M40V800 at 10 and 55 minutes after injection were noninferior to those produced by $\mathrm{M} 20$.

\section{CONCLUSIONS AND CLINICAL RELEVANCE}

Results suggested coadministration with vatinoxan did not substantially diminish visceral antinociceptive effects of medetomidine when plasma dexmedetomidine concentrations were equivalent to those produced by medetomidine alone. For somatic antinociception, noninferiority of treatments was detected at some time points. (Am J Vet Res 2020;81:299-308)
$\mathbf{M}$ edetomidine, a racemic (50:50) mixture of dexmedetomidine and levomedetomidine, is a potent and widely used veterinary sedative with some antinociceptive activity. ${ }^{1}$ The clinical use of medetomidine is currently limited to healthy animals be-

\begin{tabular}{|c|c|}
\hline ABBRE & TIONS \\
\hline $\mathrm{AUC}_{10-60}$ & $\begin{array}{l}\text { Area under the plasma concentration-versus- } \\
\text { time curve from } 10 \text { to } 60 \text { minutes } \\
\text { after drug administration }\end{array}$ \\
\hline $\mathrm{Cl}$ & Confidence interval \\
\hline CVP & Central venous blood pressure \\
\hline $\mathrm{HR}$ & Heart rate \\
\hline M20 & Medetomidine $(20 \mu \mathrm{g} / \mathrm{kg})$ \\
\hline M20V400 & $\begin{array}{l}\text { Medetomidine }(20 \mu \mathrm{g} / \mathrm{kg}) \text { and vatinoxan } \\
(400 \mu \mathrm{g} / \mathrm{kg})\end{array}$ \\
\hline M40V800 & $\begin{array}{l}\text { Medetomidine }(40 \mu g / \mathrm{kg}) \text { and vatinoxan } \\
(800 \mu \mathrm{g} / \mathrm{kg})\end{array}$ \\
\hline
\end{tabular}

cause of undesirable cardiovascular effects that could be harmful in some patients. After administration of racemic medetomidine, exposure to dexmedetomidine creates both the desired and undesired drug effects, as levomedetomidine is pharmacologically inert. ${ }^{2}$ Medetomidine produces sedation by activating $\alpha_{2}$-adrenoceptors located in the locus coeruleus in the brainstem; the same mechanism also inhibits norepinephrine release from centrally and peripherally located noradrenergic nerve endings. ${ }^{3}$ The antinociceptive effects of this drug are believed to be mediated by spinal $\alpha_{2}$-adrenoceptors located in the dorsal horn of the spinal cord and supraspinally by the locus coeruleus. ${ }^{4,5}$ Medetomidine also has important direct effects on the cardiovascular system. ${ }^{6,7}$ When administered IV, medetomidine increases arte- 
rial blood pressure ${ }^{6,8}$ through vasoconstriction mediated by activation of postsynaptic $\alpha_{2 \mathrm{~B}}$-adrenoceptors located on vascular smooth muscle cells,,${ }^{9,10}$ leading to increased systemic vascular resistance. ${ }^{7,11-13}$ The increase in arterial blood pressure partly explains the bradycardia and bradyarrhythmias associated with $\alpha_{2}$-adrenoceptor agonist administration; inhibition of sympathetic nervous system activity also contributes to these findings. ${ }^{6,8,12,14}$ Increased systemic vascular resistance and the marked decrease in HR that accompanies it are in turn responsible for the observed reduction in cardiac output.,12 These negative cardiovascular effects are evident at low doses. ${ }^{7}$ The magnitude and duration of the sedation and antinociception provided by medetomidine are also dose dependent, and the adverse effects increase with dose.,14,15

Vatinoxan, previously known as MK-467 and $\mathrm{L}-659^{\prime} 066$, is an $\alpha_{2}$-adrenoceptor antagonist that poorly penetrates the blood-brain barrier, at least in rats and marmosets, allowing selective blocking of $\alpha_{2^{-}}$ adrenoceptors in peripheral tissues. ${ }^{16}$ The peripheral selectivity of vatinoxan has also been recently found in dogs. ${ }^{17}$ Vatinoxan has been used experimentally and clinically to prevent or attenuate the undesired peripheral effects of medetomidine and dexmedetomidine in dogs, ${ }^{18-22}$ and reductions in the unwanted effects of the latter drugs are dose dependent. ${ }^{18,21}$ Centrally mediated dexmedetomidine-induced sedation is not substantially reversed by the administration of vatinoxan in dogs, ${ }^{19,23}$ although the duration of sedation is slightly shortened.$^{24}$ The modest reduction in the duration of sedation observed after vatinoxan administration probably results from decreased plasma dexmedetomidine concentrations ${ }^{24,25}$ due to increased cardiac output, resulting in higher clearance and increased volume of distribution of dexmedetomidine. ${ }^{25}$ Vatinoxan has also been shown to reduce the somatic antinociceptive potency of IV administered medetomidine in dogs, presumably through the same mechanism. ${ }^{24}$ It has been proposed ${ }^{24}$ that the reduction in sedative and antinociceptive effects should be eliminated by increasing the IV dose of medetomidine when vatinoxan is administered to achieve plasma concentrations of dexmedetomidine sufficient to produce sedation and analgesia. To the authors' knowledge, no studies have been performed to evaluate somatic antinociception in dogs that have equal plasma dexmedetomidine concentrations with or without vatinoxan, and although results of 1 study $^{26}$ revealed no evidence that vatinoxan abolishes the antinociceptive effect of dexmedetomidine after IV administration in rats used to study visceral pain, the possible impact of vatinoxan on medetomidine-induced visceral antinociception in dogs has not been assessed.

Preventing the peripherally mediated adverse effects of $\alpha_{2}$-adrenoceptor agonists with vatinoxan would be useful only if it did not diminish the sedation and antinociception provided by the agonist drug. The purpose of the study reported here was to determine whether concurrent vatinoxan administration influences the antinociceptive efficacy of medetomidine in dogs when administered at doses that provide circulating dexmedetomidine concentrations similar to those produced by medetomidine alone. We aimed to compare the magnitude of medetomidine's sedative, somatic antinociceptive, and visceral antinociceptive effects with and without concomitant vatinoxan administration. On the basis of previous results, ${ }^{24,25}$ we anticipated that plasma dexmedetomidine concentrations resulting from a given IV dose of medetomidine could be achieved when vatinoxan was coadministered IV with a higher dose of medetomidine. Our hypothesis was that medetomidine plus vatinoxan would have an antinociceptive and sedative efficacy similar to that achieved with medetomidine alone when plasma concentrations of dexmedetomidine were equal or nearly equal to those resulting from the latter treatment.

\section{Materials and Methods}

\section{Dogs}

Eight healthy purpose-bred Beagles approximately 1.5 years of age ( 2 females and 6 males; all neutered a few months prior to enrollment) were used in the study. Mean \pm SD body weight of the dogs was $13.4 \pm$ $0.9 \mathrm{~kg}$. All animals were considered healthy on the basis of physical examination findings by a veterinarian and results of a CBC and routine serum biochemical analysis.

Dogs were housed in 1 group and fed commercially available dog food twice daily with fresh water freely available. On each study day, food was withheld for $\geq 8$ hours prior to instrumentation and drug administration, and dogs were fed at the end of each experiment. All dogs were retired from research and adopted out after the study. The study protocol was approved by the Finnish National Animal Ethics Committee (license No. ESAVI/7187/04.10.03/2012).

\section{Drugs and experimental design}

The study was designed as a randomized $3 \times 3$ crossover trial. The order of treatments was randomized by use of numbered pieces of paper; the Williams design was used for the first 6 dogs, and treatment order for the remaining 2 dogs was randomly picked from the same design. Each dog received 3 treatments of medetomidine hydrochloride ${ }^{\mathrm{a}}$ alone or with vatinoxan hydrochloride $^{\mathrm{b}}$ (M20, M20V400, and M40V800), with intervals of $\geq 2$ weeks between treatments. The amount of vatinoxan was adjusted when the dose of medetomidine was increased to keep the medetomidine-to-vatinoxan concentration ratio consistent between the latter 2 treatments. The 2 drugs were mixed in the same syringe immediately prior to use. The drug combination was diluted with sterile saline $(0.9 \% \mathrm{NaCl})$ solution to achieve a total volume of $10 \mathrm{~mL}$.

At the start of each experimental session, a 20-gauge catheter ${ }^{c}$ was inserted into the left or right 
cephalic vein, and dogs were preoxygenated (100 $\mathrm{mL} / \mathrm{kg} / \mathrm{min}$ for $\geq 3$ minutes) via a tight-fitting face mask. For instrumentation, general anesthesia was induced by IV administration of propofol ${ }^{\mathrm{d}}$ to effect; the mean \pm SD dose required for tracheal intubation was $6.9 \pm 1.1 \mathrm{mg} / \mathrm{kg}$. Anesthesia was maintained with isoflurane $e^{\mathrm{e}}$ in oxygen administered through a circle breathing system, and dogs were allowed to breathe spontaneously. During instrumentation, acetated Ringer solution was administered IV at a rate of $4 \mathrm{~mL} /$ $\mathrm{kg} / \mathrm{h}$. Three ECG electrodes were placed, and dogs were covered with a blanket; rectal temperature was maintained between $36^{\circ} \mathrm{C}$ and $38^{\circ} \mathrm{C}$ with an electric heating pad. Following percutaneous infiltration of approximately $1 \mathrm{~mL}$ of $2 \%$ lidocaine hydrochloride, ${ }^{f}$ dogs were instrumented with a central venous catheterg placed via the left or right jugular vein. The catheter was measured so that the tip extended to the second rib, and correct positioning of the distal port was confirmed by observation of a typical CVP waveform. After instrumentation, isoflurane administration and IV fluid administration were discontinued, and dogs were monitored through extubation and recovery. $A \geq 60$-minute recovery interval was used to ensure appropriate recovery before recording of baseline assessments (immediately prior to study treatment administration), which included measurement of CVP and HR, sedation scoring, and measurement of somatic and visceral pressures used to evaluate nociception as subsequently described. After baseline data were obtained, the assigned study treatment was injected via the cephalic venous catheter over a 10 -second period, dogs were placed in lateral recumbency on the electric heating pad, and the infusion of acetated Ringer solution was reinitiated $(4 \mathrm{~mL} / \mathrm{kg} / \mathrm{h}, \mathrm{IV})$.

The central venous catheter was used for blood sample collection and for CVP measurements by use of a precalibrated pressure transducer ${ }^{\mathrm{h}}$ and a multiparameter monitor. ${ }^{\text {i }}$ The sternum was used as the zero reference point for CVP measurements, and HR was recorded from the ECG results. The CVP and HR measurements were obtained before performing other evaluations at baseline and 5, 10, 15, 25, 30, 35, 50, 55 , and 60 minutes after study drug injection.

Sedative and analgesic effects of the study treatments were determined by 1 researcher who was unaware of treatment assignment $(\mathrm{VH})$. Sedation scoring was performed at baseline and repeated 5, 25 , and 50 minutes after injection of the study treatment, with a composite sedation score determined as described in a previous study ${ }^{23}$; scores ranged from a minimum of 0 (no sedation) to a maximum of 16 (no palpebral reflex, tongue relaxed, and no reaction to surroundings).

Somatic nociception measurement was performed at baseline and 10, 30, and 55 minutes after study drug injection by the application of a standardized nociceptive force stimulus to the nail bed of a digit on the nondependent hind limb with an electronic algesimeter (ie, toe pinch), with gradually in- creasing force as previously described..$^{27,28}$ Visceral nociception measurement was completed at baseline and 15,35 , and 60 minutes after study drug injection, as previously described ${ }^{29,30}$; briefly, an anorectal balloon catheter ${ }^{\mathrm{k}}$ was gently inserted into the rectum to a depth of approximately 5 to $8 \mathrm{~cm}$, the balloon was gradually inflated, and the pressure exerted by the balloon on the visceral mucosa was measured with a mercury manometer. ${ }^{1}$ During nociception testing, the dogs' responses to the stimuli (limb withdrawal, head lift, vocalization, tensing of abdominal muscles, or $>10 \%$ increase in HR) were closely monitored. Following a response to the stimulus, the test was immediately discontinued (ie, the toe pinch was withdrawn or the balloon was deflated), and the force or pressure readings were recorded. To prevent tissue damage, stimulation was also discontinued if a predetermined maximum pressure reading was reached and the dog did not respond. The cutoff force for the toe pinch was $800 \mathrm{~g} / \mathrm{mm}^{2}\left(7.845 \mathrm{~N} / \mathrm{mm}^{2}\right)$ as recommended by the manufacturer, and the maximum inflation pressure applied with the rectal balloon was 1.5 times the baseline response pressure measured at the beginning of each experiment.

Blood samples $(5 \mathrm{~mL})$ were collected through the central venous catheter prior to each evaluation (ie, at baseline and 5, 10, 15, 25, 30, 35, 50, 55, and 60 minutes after injection of study drugs). The blood samples were centrifuged at $3,000 \times g$ for $15 \mathrm{~min}$ utes, and collected plasma was frozen at $-20^{\circ} \mathrm{C}$ until measurement of dexmedetomidine and vatinoxan concentrations.

At the end of each experiment, all catheters were removed. The dogs received a single $0.2-\mathrm{mg} / \mathrm{kg}$ dose of meloxicam, ${ }^{\mathrm{m}} \mathrm{SC}$, and the effects of medetomidine were reversed by IM administration of atipamezole hydrochloride, ${ }^{\mathrm{n}}$ (given according to the dose of medetomidine administered; $0.1 \mathrm{mg} / \mathrm{kg}$ for dogs that had received M20 or M20V400 and $0.2 \mathrm{mg} / \mathrm{kg}$ for dogs that had received M40V800).

\section{Plasma drug concentration analysis}

Concentrations of dexmedetomidine and vatinoxan in canine plasma were analyzed with high-performance liquid chromatography-tandem mass spectrometry as previously described. ${ }^{24}$ The analytical methods were validated for range, precision, accuracy, carryover, interference of analytes and internal standards, matrix effects, and analyte stability to comply with regulatory guidance. ${ }^{31}$ Reference standards were used for dexmedetomidine, ${ }^{\circ}$ and the linear concentration range for each enantiomer of medetomidine was from 0.075 to $10 \mathrm{ng} / \mathrm{mL}$. The interassay accuracy of the quality control samples (at concentrations of $0.15,1.0$, and $8 \mathrm{ng} / \mathrm{mL}$ ) ranged from $99.4 \%$ to $103.0 \%$ for dexmedetomidine. Reference standards for vatinoxan were used, ${ }^{\mathrm{p}}$ and the linear range of the assay was from 10 to $900 \mathrm{ng} / \mathrm{mL}$. The interassay accuracy of the quality control samples (at concentrations of 30,400 , and $740 \mathrm{ng} / \mathrm{mL}$ ) ranged from $97.4 \%$ 
to $109.1 \%$. Intraassay coefficients of variation were < $8 \%$ for both analytes at all 3 concentrations, and < $15 \%$ at the lower limit of quantitation $(0.075 \mathrm{ng} / \mathrm{mL}$ for dexmedetomidine and $10 \mathrm{ng} / \mathrm{mL}$ for vatinoxan).

\section{Statistical analysis}

Normality assumptions were checked for all response variables ( $\mathrm{HR}, \mathrm{CVP}$, and $\mathrm{AUC}_{10-60}$ values for dexmedetomidine concentration, and areas under the measurement-versus-time curve over the same interval for sedation scores and visceral and somatic pressure measurements) with Kolmogorov-Smirnov tests. ${ }^{q}$ Only CVP measurements were normally distributed. The visceral and somatic nociception measurements and sedation scores obtained after injection of study drugs were compared with the corresponding baseline measurements and among the 3 treatments within time points; this analysis was performed for related samples with the Friedman 2-way ANOVA by ranks, followed by Bonferroni post hoc corrections when appropriate. ${ }^{\mathrm{q}}$

The sedation scores and baseline-adjusted visceral pressure measurements (calculated because the maximum balloon pressure applied depended on the baseline measurement) were further analyzed to assess noninferiority of medetomidine plus vatinoxan at each dose combination, compared with medetomidine alone for eliciting desired effects, and plasma drug concentration data were analyzed for equivalence calculation and comparisons of area under the curve between treatments. ${ }^{r}$ Only time points $\geq 10$ minutes after study drug administration were included in the analyses to ensure adequate drug distribution. The trapezoidal method was used to calculate the $\mathrm{AUC}_{10-60}$ for dexmedetomidine concentration and areas under the curve for sedation score and visceral pressures for each dog individually. Logarithmic (base 10) transformation was applied to area-underthe-curve values to normalize their distributions (confirmed with a Kolmogorov-Smirnov test). Treatment differences and 95\% CIs were transformed back to the original scale and reported as the geometric mean ratio. For intertreatment comparison of plasma dexmedetomidine concentrations, the reference limits for equivalence were set at 0.8 and 1.25 , similar to procedures used in bioequivalence studies. ${ }^{32}$ For intertreatment comparison of sedation scores and visceral pressures, the reference limits for noninferiority were also set at 0.8 and $1.25 .{ }^{32}$ Equivalence of drug concentrations or noninferiority of treatments was accepted when the $95 \% \mathrm{CI}$ of the geometric mean ratio was within this reference range.

Because the maximum value for somatic nociceptive force measurements could not be defined for each dog (owing to use of a maximum cutoff force of $800 \mathrm{~g} / \mathrm{mm}^{2}$ ), the area-under-the-curve method could not be reliably used for tolerance of somatic nociception, and nonparametric 95\% CIs were calculated for median differences between the treatments on the basis of the Wilcoxon 1-sample test statistic. ${ }^{r}$ The noninferiority margin was set at $-20 \%$ of the median value for dogs that received M20 $\left(700 \mathrm{~g} / \mathrm{mm}^{2}\right)$, which was considered a clinically acceptable difference.

The differences in changes of HR and CVP from baseline ( 0 minutes $=$ immediately prior to injection of study drugs) among treatments were evaluated with repeated-measures ANCOVA models. ${ }^{r}$ Each model included the main effects of treatment and time point, 2-way interactions of treatment by time point, and a baseline covariate as fixed effects and included the main effect of $\mathrm{dog}$ and the 2-way interaction of dog by time point as random effects. For HR, square root transformation was used to normalize the distribution. Treatment differences and within-treatment changes with 95\% CI were calculated from the same models with contrasts.

All analyses were performed with commercially available software. ${ }^{\mathrm{q}, \mathrm{r}}$ For all comparisons, values of $P<0.05$ were considered significant.

\section{Results}

One dog removed its central venous catheter after instrumentation and did not receive the M40V800 treatment. Another dog became slightly agitated and defecated loose feces together with a plastic foreign body 30 minutes after treatment administration of M20V400, and visceral nociceptive testing was discontinued for the animal at that time.

The log-transformed mean \pm SD plasma dexmedetomidine and vatinoxan concentrations are depicted (Figure I). The mean resulting plasma dexmedetomidine concentrations after M20 administration ranged from 9.31 to $2.73 \mathrm{ng} / \mathrm{mL}$, those after M20V400 administration ranged from 4.62 to $1.45 \mathrm{ng} /$ $\mathrm{mL}$, and those after M40V800 ranged from 9.23 to $2.78 \mathrm{ng} / \mathrm{mL}$; the highest values were measured at 10 minutes, and the lowest values were measured at 60 minutes (the 5-minute values were not included in the statistical analysis). The dexmedetomidine concentrations were equivalent between M40V800 and $\mathrm{M} 20$, as confirmed with the $\mathrm{AUC}_{10-60}$ comparisons (geometric mean ratio, 0.92 [95\% CI, 0.82 to 1.04]). After M20V400 administration, plasma dexmedetomidine concentrations were approximately halved, compared with those after M20 administration, at all analyzed time points, and the plasma concentrations were not equivalent between M20V400 and M20 (geometric mean ratio, 0.46 [95\% CI, 0.40 to 0.51$]$ ). The concentrations of vatinoxan in plasma were linearly proportional to the administered doses.

Median and range maximum rectal balloon inflation pressures (visceral nociception measurements) and the proportion of dogs that did not react at the maximum cutoff pressure after each treatment are shown (Table I). The M20 and M40V800 treatments were each associated with significantly increased visceral pressure tolerance, compared with that at baseline in the same experiment, 15 and 35 minutes after treatment. No significant differences from baseline measurements were found after administration 
of M20V400. When maximum visceral pressure measurements within time points were compared among the 3 treatments, no significant difference was found. Comparisons of areas under the measurement-versustime curve from 10 to 60 minutes after study drug administration confirmed that visceral antinociception with M40V800 was noninferior to that produced by M20 administration (geometric mean ratio, 0.96 [95\% CI, 0.84 to 1.1$]$ ). Noninferiority of visceral antinociception with M20V400 could not be shown (geometric mean ratio, 0.90 [95\% CI, 0.79 to 1.0$]$ ]).

Median and range maximum force applied to the nail bed (somatic nociception measurements) and the number of animals that did not react to the predetermined cutoff force are reported (Table 2). Somatic nociceptive force tolerance was significantly in- creased at 10 and 30 minutes (but not at 55 minutes) after all treatments, compared with the respective baseline measurements. When somatic nociception measurements within time points were compared among the 3 treatments, no significant difference was found. With the noninferiority margin set at $-20 \%$, somatic antinociception with M20V400 was noninferior to that achieved with M20 10 minutes after treatment administration, and somatic antinociception with M40V800 was noninferior to that resulting from M20 at 10 and 55 minutes (but not 30 minutes) after treatment administration.

The median and range sedation scores are summarized (Table 3). Compared with the respective baseline values, the scores obtained after all treatments were significantly higher (indicating signifi-
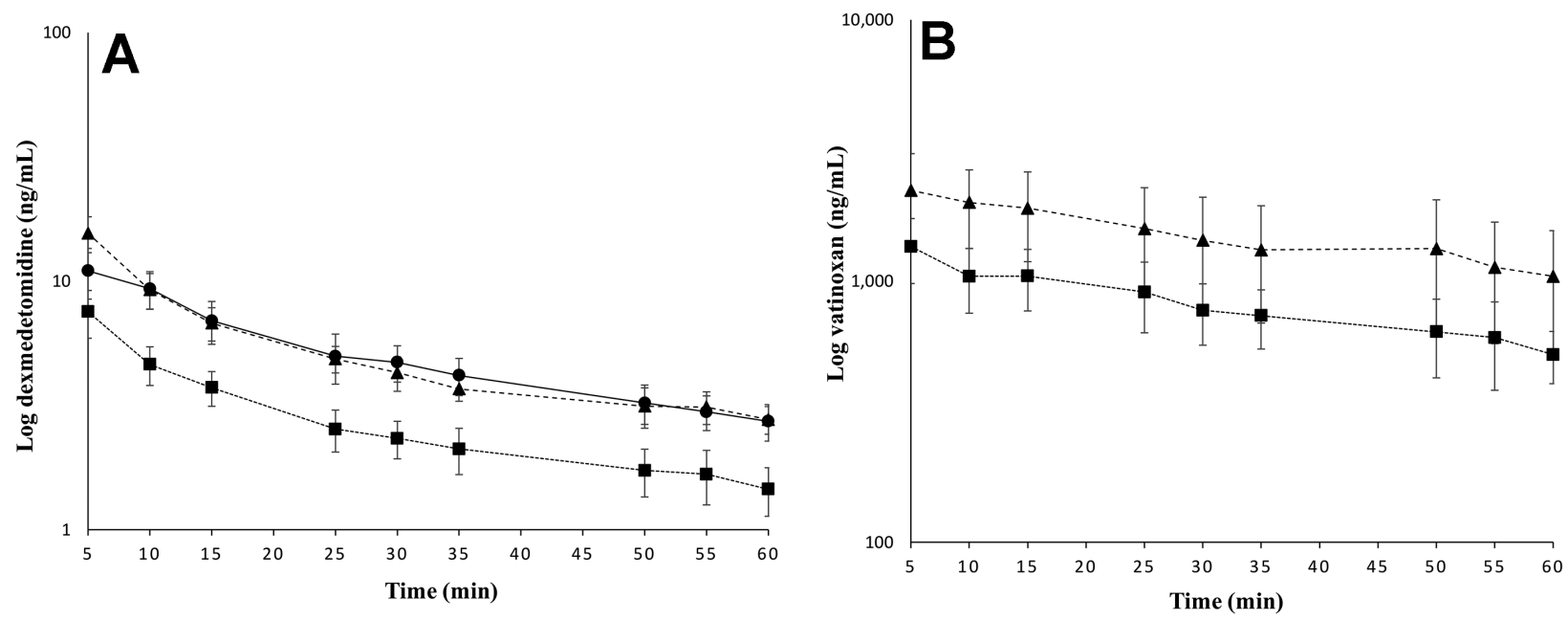

Figure I-Semilogarithmic plots of the mean \pm SD plasma concentrations of dexmedetomidine (A) and vatinoxan (B) versus time in 8 healthy Beagles from 5 to 60 minutes after IV administration (time 0) of M20 (circles), M20V400 (squares), or M40V800 (triangles) sedative treatments in a randomized $3 \times 3$ crossover-design study. Combinations of medetomidine and vatinoxan were mixed in the same syringe; all study drugs were diluted with sterile saline $(0.9 \% \mathrm{NaCl})$ solution to a total volume of $10 \mathrm{~mL}$ and injected IV over 10 seconds. There was a washout period of $\geq 14$ days between treatments. Data for M40V800 represent results for 7 dogs (I dog did not receive the treatment).

Table I-Median (range) maximum visceral pressure measurements in 8 healthy Beagles that received M20, M20V400, or M40V800 treatment in a randomized $3 \times 3$ crossover-design study and the proportion of dogs that did not react to the stimulus up to and including the maximum cutoff pressure applied ( 1.5 times the baseline pressure for the same dog during each experiment).

\begin{tabular}{|c|c|c|c|c|c|c|}
\hline \multirow[b]{2}{*}{ Time (min) } & \multicolumn{2}{|c|}{ M20 } & \multicolumn{2}{|c|}{ M20V400 } & \multicolumn{2}{|c|}{ M40V800 } \\
\hline & $\begin{array}{l}\text { Measurement } \\
(\mathrm{mm} \mathrm{Hg})\end{array}$ & $\begin{array}{l}\text { Proportion with } \\
\text { no reaction }\end{array}$ & $\begin{array}{l}\text { Measurement } \\
(\mathrm{mm} \mathrm{Hg})\end{array}$ & $\begin{array}{l}\text { Proportion with } \\
\text { no reaction }\end{array}$ & $\begin{array}{l}\text { Measurement } \\
(\mathrm{mm} \mathrm{Hg})\end{array}$ & $\begin{array}{l}\text { Proportion with } \\
\text { no reaction }\end{array}$ \\
\hline 0 (baseline) & $126(96-180)$ & - & $128(110-174)$ & - & $128(115-172)$ & - \\
\hline 15 & $187(134-250)^{*}$ & $2 / 8$ & $175(152-242)$ & $2 / 8$ & $180(140-250) *$ & $4 / 7$ \\
\hline 35 & $189(144-260)^{*}$ & $4 / 8$ & $165(120-248)$ & $\mathrm{I} / 7$ & $173(156-207)^{*}$ & $2 / 7$ \\
\hline 60 & $17 \mid(\mid 44-230)$ & $2 / 8$ & $164(120-212)$ & $2 / 7$ & $165(138-174)$ & $0 / 7$ \\
\hline
\end{tabular}

Baseline values were obtained immediately prior to injection of study drugs. Measurements were obtained by use of an anorectal balloon catheter placed in the rectum; the balloon was gradually inflated until a reaction to the stimulus (limb withdrawal, head lift, vocalization, tensing of abdominal muscles, or $>10 \%$ increase in HR) was observed by an individual blinded to the treatment administered or until the predetermined cutoff pressure was reached, at which time the balloon was immediately deflated and the last inflation pressure was recorded. One dog was excluded from testing because it did not receive M40V800 treatment, and I dog had visceral nociception testing discontinued 30 minutes after M20V400 administration because it developed loose feces with evidence of prior foreign body consumption. Values of $P<0.05$ were considered significant.

*Value is significantly different, compared with that at baseline for the same treatment.

$-=$ Not applicable. 
Table 2-Median (range) maximum somatic nociceptive force measurements for the dogs in Table I and the proportion of dogs that did not react to the stimulus up to and including the maximum cutoff force applied $\left(800 \mathrm{~g} / \mathrm{mm}^{2}\right)$.

\begin{tabular}{|c|c|c|c|c|c|c|}
\hline \multirow[b]{2}{*}{ Time (min) } & \multicolumn{2}{|c|}{ M20 } & \multicolumn{2}{|c|}{ M20V400 } & \multicolumn{2}{|c|}{ M40V800 } \\
\hline & $\begin{array}{l}\text { Measurement } \\
\left(\mathrm{g} / \mathrm{mm}^{2}\right)\end{array}$ & $\begin{array}{l}\text { Proportion with } \\
\text { no reaction }\end{array}$ & $\begin{array}{c}\text { Measurement } \\
\left(\mathrm{g} / \mathrm{mm}^{2}\right)\end{array}$ & $\begin{array}{l}\text { Proportion with } \\
\text { no reaction }\end{array}$ & $\begin{array}{l}\text { Measurement } \\
\left(\mathrm{g} / \mathrm{mm}^{2}\right)\end{array}$ & $\begin{array}{l}\text { Proportion with } \\
\text { no reaction }\end{array}$ \\
\hline 0 (baseline) & $371(202-598)$ & $0 / 8$ & $321(175-566)$ & $0 / 8$ & $440(290-704)$ & $0 / 7$ \\
\hline 10 & $800(456-800) *$ & $5 / 8$ & 800 (756-800)* & $6 / 8$ & $800(588-800) *$ & $4 / 7$ \\
\hline 30 & $800(574-800)^{*}$ & $5 / 8$ & $725(572-800)^{*}$ & $2 / 8$ & $789(506-800)^{*}$ & $3 / 7$ \\
\hline 55 & $604(322-800)$ & $2 / 8$ & $520(430-800)$ & $1 / 8$ & $601(344-716)$ & $0 / 7$ \\
\hline
\end{tabular}

Measurements were obtained by application of gradually increasing force (delivered by an electronic algesimeter) to the nail bed of a digit on the nondependent hind limb of dogs in lateral recumbency. The test was immediately discontinued when a reaction to the stimulus was observed by an individual blinded to the treatment administered or when the maximum cutoff force was reached and the last force applied was recorded.

See Table I for remainder of key.

Table 3-Median (range) sedation scores for the dogs in Table I.

\begin{tabular}{lccc} 
Time (min) & $\begin{array}{c}\text { M20 } \\
(\mathbf{n = 8 )}\end{array}$ & $\begin{array}{c}\text { M20V400 } \\
(\mathbf{n}=\mathbf{8})\end{array}$ & $\begin{array}{c}\text { M40V800 } \\
(\mathbf{n}=\mathbf{7})\end{array}$ \\
\hline 0 (baseline) & $0(0-0)$ & $0(0-0)$ & $0(0-0)$ \\
5 & $14(8-16)^{*}$ & $12(9-14)^{*}$ & $13(8-15)^{*}$ \\
25 & $13.5(11-15)^{*}$ & $14(11-15)^{*}$ & $14(10-16)^{*}$ \\
50 & $8.5(8-10)^{*}$ & $7.5(6-12)^{*}$ & $9(8-12)^{*}$
\end{tabular}

Sedation was scored by I investigator who was blinded to the treatment administered; the scoring method was adapted from another source, ${ }^{23}$ and possible scores ranged from 0 (no sedation) to 16 (maximum sedation; no palpebral reflex, tongue relaxed, no reaction to surroundings).

See Table I for remainder of key.

cantly greater sedation) at all evaluated time points. When sedation scores were compared among the 3 treatments at each of the assessment time points, no significant differences were detected. Noninferiority of M40V800 and of M20V400 relative to M20 for sedative effects was confirmed by comparison of areas under the measurement-versus-time curves from 10 to 60 minutes after the study drug injection: for M40V800, the geometric mean ratio was 1.01 (95\% CI, 0.88 to 1.17 ), and for M20V400, the geometric mean ratio was 0.97 ( $95 \% \mathrm{CI}, 0.84$ to 1.12$)$.

Measurements of HR were significantly higher and measurements of CVP were significantly lower at each time point after administration of M20V400 or M40V800, compared with the results for M20 treatment (Table 4). The HR measurements were significantly lower and CVP measurements were significantly higher, compared with the respective baseline values at all postinjection time points for M20 treatment. No significant differences in HR or CVP were detected at any time point between M20V400 and M40V800.

\section{Discussion}

In the present study, we compared sedative and antinociceptive effects of 2 dose combinations of medetomidine and vatinoxan with those of medetomidine alone. After adequate drug distribution, which appeared to require $>5$ minutes on the basis of the plasma concentration curve and data from previ- ous studies, including a study ${ }^{25}$ of dogs, M40V800 produced plasma dexmedetomidine concentrations equivalent to those resulting from M20 administration, and plasma concentrations remained similar between these 2 treatments throughout the investigation (from 10 to 60 minutes). When M20V400 was administered, the resulting plasma dexmedetomidine concentrations were considerably lower at approximately half those of M20 throughout the evaluation period, probably owing to the vatinoxan-induced reduction in initial vasoconstriction and preserved cardiac output (which were indirectly suggested by lower CVP and higher HR), leading to higher clearance from increased perfusion of elimination organs and a larger volume of dexmedetomidine distribution. ${ }^{24,25}$ As expected, once plasma concentrations of dexmedetomidine were similar between the 2 treatments, the sedation and visceral antinociception produced by M40V800 was noninferior to that produced by M20 at all evaluated time points, with similar results for somatic antinociception at 10 and 55 minutes. The sedation achieved with M20V400 was also noninferior to that resulting from M20, despite the lower plasma dexmedetomidine concentrations. Unimpaired sedative effects were probably seen because even the reduced dexmedetomidine plasma concentrations after M20V400 injection were high enough to induce maximal sedative effects; therefore, the increased medetomidine dose (and plasma dexmedetomidine concentration) did not further augment the effect.

We assessed somatic antinociception effects of the treatments by applying a standardized nociceptive force to the nail bed, which was stopped when a predetermined maximum force was reached or when the dog withdrew the limb or showed any signs of sympathetic stimulation or discomfort. Limb withdrawal is a spinally mediated response to nociception, ${ }^{4}$ and the antinociceptive effect of dexmedetomidine is believed to be mediated mainly by activation of $\alpha_{2}$-adrenoceptors in the spinal cord. ${ }^{33}$ In another study, ${ }^{24}$ when $10 \mu \mathrm{g}$ of medetomidine/kg was administered with $250 \mu \mathrm{g}$ of vatinoxan/kg IV to dogs, the anticipated plasma dexmedetomidine concentration was halved. The resulting plasma dexmedetomidine concentration did not exceed $1.9 \mathrm{ng} / \mathrm{mL},{ }^{24}$ which is 
Table 4-Median (range) HR and CVP measurements for the dogs in Table I.

\begin{tabular}{|c|c|c|c|c|c|c|}
\hline \multirow[b]{2}{*}{ Time (min) } & \multicolumn{2}{|c|}{$M 20(n=8)$} & \multicolumn{2}{|c|}{ M20V400 $(n=8)$} & \multicolumn{2}{|c|}{ M40V800 $(n=7)$} \\
\hline & $\begin{array}{c}\text { HR } \\
\text { (beats/min) }\end{array}$ & $\begin{array}{c}\text { CVP } \\
(\mathrm{mm} \mathrm{Hg})\end{array}$ & $\begin{array}{c}\text { HR } \\
\text { (beats/min) }\end{array}$ & $\begin{array}{c}\text { CVP } \\
(\mathrm{mm} \mathrm{Hg})\end{array}$ & $\begin{array}{c}\text { HR } \\
\text { (beats/min) }\end{array}$ & $\begin{array}{c}\text { CVP } \\
(\mathrm{mm} \mathrm{Hg})\end{array}$ \\
\hline 0 (baseline) & 86 (74 to II9) & I.5 (-I to 3$)$ & 94 (72 to 127) & $2.0(0$ to 5$)$ & $102(76$ to I53) & $2.0(0$ to 3$)$ \\
\hline 5 & 34.5 (28 to 44$)^{*}$ & $8.5(5 \text { to } \mathrm{II})^{*}$ & $67(44$ to 88$) \dagger$ & $3.5(-1$ to 7$) \dagger$ & $72(60$ to 100$) \dagger$ & $3.0(I$ to 5$) \dagger$ \\
\hline 10 & 32 (24 to 44$)^{*}$ & 7.5 (5 to II)* & $76(25$ to 108$) \dagger$ & $4.0(-3$ to 6$) \dagger$ & $76(61$ to 92$) t$ & $2.0(I$ to 4$) \dagger$ \\
\hline 15 & 38 (30 to 48$)^{*}$ & $7.0(4 \text { to } 10)^{*}$ & $69(55$ to 110$) \dagger$ & $3.0(-3$ to 6$) \dagger$ & $75(63$ to 88$) \dagger$ & 3.0 (I to 4$) \dagger$ \\
\hline 25 & $36(28 \text { to } 48)^{*}$ & $5.5(3 \text { to } 10)^{*}$ & $70(62$ to 112$) \dagger$ & $3.0(-2$ to 6$) \dagger$ & $75(60$ to 88$) \dagger$ & $2.0(1$ to 3$) \dagger$ \\
\hline 30 & 36 (36 to 48$)^{*}$ & 5.5 (2 to 9$)^{*}$ & $68(57$ to 92$) \dagger$ & $2.5(-3$ to 6$) \dagger$ & $72(59$ to 84$) \dagger$ & $1.0(0$ to 3$) \dagger$ \\
\hline 35 & $37(24 \text { to } 44)^{*}$ & 5.5 (I to 9$)^{*}$ & $66(56$ to 84$) \dagger$ & $2.5(-2$ to 6$) \dagger$ & $72(60$ to 76$) \dagger$ & $2.0(0$ to 3$) \dagger$ \\
\hline 50 & $34(32 \text { to } 44)^{*}$ & 5.0 (2 to 9$)^{*}$ & $62(44$ to 79$) \dagger$ & $2.5(-3$ to 5$) \dagger$ & $64(56$ to 76$) \dagger$ & $2.0(-1$ to 3$) \dagger$ \\
\hline 55 & $40(28 \text { to } 42)^{*}$ & 4.0 (2 to 9$)^{*}$ & $59(56$ to 80$) \dagger$ & $2.0(-2$ to 6$) \dagger$ & 65 (52 to 72$) \dagger$ & $1.0(-2$ to 3$) \dagger$ \\
\hline 60 & $40(24 \text { to } 44)^{*}$ & $4.5(2 \text { to } 8)^{*}$ & $64(52$ to 138$) \dagger$ & $2.0(-4$ to 4$) \dagger$ & $64(52$ to 72$) \dagger$ & $2.0(-1$ to 3$) \dagger$ \\
\hline
\end{tabular}

†Within a timepoint, value is significantly different from that for the same measurement after M20 administration.

See Table I for remainder of key.

the mean concentration considered to be required for antinociception in dogs. ${ }^{34}$ This led to a significant reduction in medetomidine-induced somatic antinociception (measured by squeezing the nail bed with hemostats), compared with results when the same dose of medetomidine was given alone, and sedation was achieved but shortened relative to that from medetomidine alone. ${ }^{24}$ Those findings corresponded with previous findings that higher plasma dexmedetomidine concentrations are required for antinociception than for sedation. ${ }^{14,34}$ The mean plasma dexmedetomidine concentration associated with sedation in dogs is $\geq 0.533 \mathrm{ng} / \mathrm{mL}^{34}$ In our study, plasma dexmedetomidine concentrations persisted above the previously reported analgesic concentration (1.9 ng/ $\mathrm{mL})^{34}$ in all dogs throughout the investigation period after the M20 or M40V800 treatment. After M20V400 administration, the mean plasma dexmedetomidine concentrations exceeded $1.9 \mathrm{ng} / \mathrm{mL}$ for the first 35 minutes, after which they decreased gradually but remained well above the concentration needed for sedation ${ }^{34}$ until the end of our study.

Visceral antinociception was assessed by inflating a purpose-made anorectal balloon catheter inside the rectum and measuring pressures inside the balloon. We chose rectal distension for assessment of visceral pain because the rectal catheter is easy to insert, the results are quantifiable, and the method is minimally invasive; the procedure is commonly used to assess visceral nociception in rats, dogs, horses, and people. ${ }^{26,29,30,35-38}$ We measured the pressure within the rectal balloon because in human subjects, visceral pain sensation is reportedly related to pressure rather than volume of distension. ${ }^{39}$ We found that M40V800 was noninferior to M20 in the provision of visceral antinociception. Due to variations in baseline measurements even within the same dog, we decided to end testing at a maximum inflation pressure of 1.5 times the baseline pressure (or less, if the dog responded earlier). Although it would have been simpler to use a fixed maximum inflation pressure, we made this choice to avoid tissue damage. In addition to the ethical consideration to do no harm, any degree of tissue injury could have interfered with subsequent measurements because the dogs served as their own controls in the crossover-design study. Other researchers have used a similar approach: results of another visceral analgesia study ${ }^{29}$ in dogs revealed that rectal balloon inflation pressures of 120 to $190 \mathrm{~mm} \mathrm{Hg}$ were required to elicit a response in awake dogs (pretreatment control values). After butorphanol administration, antinociception was assumed to be present if the pressure required to elicit a response was higher than the pretreatment values. The investigators of that study ${ }^{29}$ expressed the inflation pressures as percentage increases from the control pressures; exact numeric values were not reported, but the highest pressures used were roughly $40 \%$ higher than the control pressures. ${ }^{29}$ The reported control pressures and the percentage change in pressure tolerance after butorphanol administration ${ }^{29}$ were similar to those measured in our study.

Overall, our results supported the assumption ${ }^{24}$ that the previously described decrease in antinociceptive efficacy and the shorter duration of sedation in dogs that received vatinoxan in addition to medetomidine were more likely caused by lower plasma dexmedetomidine concentrations than the ability of vatinoxan to permeate the canine blood-brain barrier to any clinically relevant extent. It has been shown that vatinoxan injection in rats and marmosets produces minimal brain concentrations of the drug, ${ }^{16}$ and recent results from our study group suggest that the same is true for dogs. ${ }^{17}$ In dogs, however, vatinoxan has been shown to increase the minimum alveolar concentration of sevoflurane. ${ }^{40}$ The spinal cord is considered important in suppressing movement in response to noxious stimulation under inhalation anesthesia and therefore in the measurement of minimum alveolar concentration ${ }^{41-43}$; if vatinoxan increases the minimum alveolar concentration of sevoflurane, it is possible that some spinal cord penetration occurs. ${ }^{40}$ In the study reported here, no significant differences in spinally mediated somatic antinociception were found among the 3 treatments, and somatic antinociception resulting from M40V800 administration was not inferior to that from M20 at 
10 and 55 minutes after administration. In addition, vatinoxan administration produces low spinal cord-toblood concentration ratios of the drug in dogs, indicating that only a very small fraction of vatinoxan is able to permeate into the CNS. ${ }^{17}$ However, the brain and spinal cord concentrations of vatinoxan sufficient to cause measurable CNS effects are not known.

Although the extents of sedation and antinociception provided by medetomidine are generally considered to be dose dependent and increasing plasma dexmedetomidine concentrations will initially result in more pronounced effects, a so-called ceiling effect at a certain concentration is reached in dogs, after which increasing the dose no longer increases the magnitude of sedation but increases the duration of this effect. ${ }^{14,34}$ However, both sedation ${ }^{44}$ and antinociception $^{44,45}$ in cats are intensified with increasing plasma dexmedetomidine concentrations, suggesting that higher concentrations may be required to reach such a ceiling effect in cats than in dogs.

Clinically relevant and statistically significant decreases in HR and increases in CVP were detected after M20 administration in the present study. As previously described, ${ }^{18-21,46}$ vatinoxan administration abolished these hemodynamic changes. Both tested drug doses were sufficient to reverse the unwanted effects of medetomidine on HR and CVP.

One dog had loose feces and defecated a plastic foreign body 30 minutes after vatinoxan administration, after which the dog was excluded from further assessments of visceral antinociception. It was likely that the foreign body had irritated the bowel; however, it was also possible that vatinoxan alleviated medetomidine-induced intestinal relaxation and increased gut motility, thus leading to loosened feces. In people and horses, $\alpha_{2}$-adrenoceptor agonists have been shown to inhibit gastric emptying and reduce gut motility. ${ }^{47-49}$ Conversely, vatinoxan has been shown to antagonize detomidine-induced and romifidine-induced intestinal hypomotility in horses, ${ }^{48,49}$ and results of 1 study ${ }^{49}$ indicate abdominal discomfort, evidenced by restlessness and kicking, in 3 of 7 horses after administration of vatinoxan alone. Furthermore, loose feces were detected in one-third of client-owned dogs (9/27) that had been sedated with a medetomidine-butorphanol-vatinoxan combination for diagnostic imaging; however, in the same study, ${ }^{22}$ several dogs that received atipamezole after being sedated with a medetomidine-butorphanol combination without vatinoxan also developed loose feces. In our study, only $1 \mathrm{dog}$ was found to have loose feces, and no definitive conclusions could be drawn regarding the rate of occurrence of this possible adverse effect.

Our study had several limitations. The main limitation was that our study may have lacked statistical power to detect a significant difference in sedation scores among treatments, even when the dexmedetomidine plasma concentration was halved by coadministration of vatinoxan with the medetomidine.
A probable contributing factor was that the dexmedetomidine plasma concentration still remained high enough to induce (nearly) maximal sedation. It was also possible that our sedation scoring system, despite its frequent use in similar studies, was not sensitive enough to detect differences among the treatments. In ideal conditions, the postinjection observation period would have been longer, and the study would have included a treatment that consisted of vatinoxan alone. Also, considering that the aim of a noninferiority trial is to show that the experimental treatment is not less effective than the active control by more than the noninferiority margin, the noninferiority margin chosen should be the largest difference that can be considered clinically acceptable; however, this is a subjective judgement, and our choice of this margin was therefore a possible limitation.

As previously described, higher plasma dexmedetomidine concentrations were required for analgesia than for sedation in the present study. The addition of vatinoxan did not considerably interfere with the analgesia provided by medetomidine as long as the plasma dexmedetomidine concentrations remained greater than amounts required for antinociception. Vatinoxan also provided better cardiovascular stability (suggested by the absence of marked bradycardia and lower CVP), compared with the administration of medetomidine alone. Additional studies of vatinoxanmedetomidine combinations as a part of a balanced anesthesia protocol are warranted to verify the suitability of vatinoxan in clinical veterinary use in dogs.

\section{Acknowledgments}

Funded by Vetcare Ltd (study drugs, consumables, and plasma concentration analyses) and the Finnish Foundation of Veterinary Research (a grant for statistical analysis). Dr. Restitutti received financial support from Vetcare Ltd during the study. However, Vetcare Ltd did not have any involvement in the study design, data collection, data analysis and interpretation, or writing and publication of the manuscript. The authors declare that there were no other conflicts of interest.

The authors thank Mr. Lauri Vuorilehto for performing plasma drug concentration analyses and Dr. Mari Palviainen for technical assistance.

\section{Footnotes}

a. Dorbene, Vetcare Ltd, Mäntsälä, Finland.

b. Vetcare Ltd, Mäntsälä, Finland.

c. Terumo Europe NV, Leuven, Belgium.

d. Vetofol 1\%, Norbrook Laboratories, Newry, Northern Ireland.

e. Isoflo, Orion Pharma Ltd, Espoo, Finland.

f. Orion Oyj, Turku, Finland

g. CV-12702, Arrow International, Reading, Pa.

h. Gabarith PMSET, Becton Dickinson, Sandy, Utah

S/5 Anesthesia Monitor, GE Healthcare, Helsinki, Finland.

j. Randall-Selitto, IITC 2500 Digital Paw Pressure Meter, IITC Life Science Inc, Woodland Hills, Calif.

k. SR1B Single-use anorectal balloon/expulsion catheter, Mui Scientific, Mississauga, ON, Canada.

1. Welch Allyn UK Ltd, Buckinghamshire, England.

m. Metacam $5 \mathrm{mg} / \mathrm{mL}$, Boehringer Ingelheim Vetmedica, Ingelheim, Germany.

n. Antisedan $5 \mathrm{mg} / \mathrm{mL}$, Orion Pharma Ltd, Espoo, Finland.

o. Toronto Research Chemicals, Toronto, ON, Canada. 
p. PCAS Finland Oy, Turku, Finland.

q. SPSS statistics for Mac, version 24.0, IBM Corp, Armonk, NY.

r. SAS for Windows, version 9.3, SAS Institute Inc, Cary, NC.

\section{References}

1. Murrell JC, Hellebrekers LJ. Medetomidine and dexmedetomidine: a review of cardiovascular effects and antinociceptive properties in the dog. Vet Anaesth Analg 2005;32:117127.

2. MacDonald E, Scheinin M, Scheinin H, et al. Comparison of the behavioral and neurochemical effects of the two optical enantiomers of medetomidine, a selective alpha-2-adrenoceptor agonist. J Pharmacol Exp Ther 1991;259:848-854.

3. Correa-Sales C, Rabin BC, Maze M. A hypnotic response to dexmedetomidine, an alpha 2 agonist, is mediated in the locus coeruleus in rats. Anesthesiology 1992;76:948-952.

4. Guo TZ, Jiang JY, Buttermann AE, et al. Dexmedetomidine injection into the locus ceruleus produces antinociception. Anesthesiology 1996;84:873-881.

5. Buerkle H, Yaksh TL. Pharmacological evidence for different alpha 2-adrenergic receptor sites mediating analgesia and sedation in the rat. BrJ Anaesth 1998;81:208-215.

6. Vainio O, Palmu L. Cardiovascular and respiratory effects of medetomidine in dogs and influence of anticholinergics. Acta Vet Scand 1989;30:401-408.

7. Pypendop BH, Verstegen JP. Hemodynamic effects of medetomidine in the dog: a dose titration study. Vet Surg 1998;27:612-622.

8. Savola JM. Cardiovascular actions of medetomidine and their reversal by atipamezole. Acta Vet Scand Suppl 1989;85:3947.

9. Savola JM, Ruskoaho H, Puurunen J, et al. Evidence for medetomidine as a selective and potent agonist at alpha 2 -adrenoreceptors. J Auton Pharmacol 1986;6:275-284.

10. Link RE, Desai K, Hein L, et al. Cardiovascular regulation in mice lacking alpha2-adrenergic receptor subtypes b and c. Science 1996;273:803-805.

11. Flacke JW, Flacke WE, Bloor BC, et al. Hemodynamic effects of dexmedetomidine, an alpha 2-adrenergic agonist, in autonomically denervated dogs. J Cardiovasc Pharmacol 1990;16:616-623.

12. Bloor BC, Frankland M, Alper G, et al. Hemodynamic and sedative effects of dexmedetomidine in dog. $J$ Pharmacol Exp Ther 1992;263:690-697.

13. Flacke WE, Flacke JW, Bloor BC, et al. Effects of dexmedetomidine on systemic and coronary hemodynamics in the anesthetized dog. J Cardiotborac Vasc Anesth 1993;7:41-49.

14. Kuusela E, Raekallio M, Anttila M, et al. Clinical effects and pharmacokinetics of medetomidine and its enantiomers in dogs. J Vet Pharmacol Ther 2000;23:15-20.

15. Kuusela E, Raekallio M, Väisänen M, et al. Comparison of medetomidine and dexmedetomidine as premedicants in dogs undergoing propofol-isoflurane anesthesia. Am J Vet Res 2001;62:1073-1080.

16. Clineschmidt BV, Pettibone DJ, Lotti VJ, et al. A peripherally acting alpha-2 adrenoceptor antagonist: L-659,066. J Pharmacol Exp Ther 1988;245:32-40.

17. Honkavaara JM, Raekallio MR, Syrja PM, et al. Concentrations of medetomidine enantiomers and vatinoxan, an $\alpha_{2}$ adrenoceptor antagonist, in plasma and central nervous tissue after intravenous coadministration in dogs. Vet Anaesth Analg 2020;47:47-52.

18. Pagel PS, Proctor LT, Devcic A, et al. A novel alpha 2-adrenoceptor antagonist attenuates the early, but preserves the late cardiovascular effects of intravenous dexmedetomidine in conscious dogs. J Cardiothorac Vasc Anesth 1998;12:429434.

19. Honkavaara JM, Raekallio MR, Kuusela EK, et al. The effects of L-659,066, a peripheral alpha2-adrenoceptor agonist, on dexmedetomidine-induced sedation in dogs. Vet Anaesth Analg 2008;35:409-413.

20. Enouri SS, Kerr CL, McDonell WN, et al. Effects of a peripheral $\alpha_{2}$ adrenergic-receptor antagonist on the hemodynamic changes induced by medetomidine administration in conscious dogs. Am J Vet Res 2008;69:728-736.

21. Honkavaara JM, Restitutti F, Raekallio MR, et al. The effects of increasing doses of MK-467, a peripheral alpha(2)-adrenergic receptor antagonist, on the cardiopulmonary effects of intravenous dexmedetomidine in conscious dogs. J Vet Pharmacol Ther 2011;34:332-337.

22. Kallio-Kujala IJ, Turunen HA, Raekallio MR, et al. Peripherally acting $\alpha$-adrenoceptor antagonist MK-467 with intramuscular medetomidine and butorphanol in dogs: a prospective, randomised, clinical trial. Vet J 2018;240:22-26.

23. Restitutti F, Honkavaara JM, Raekallio MR, et al. Effects of different doses of L-659'066 on the bispectral index and clinical sedation in dogs treated with dexmedetomidine. Vet Anaestb Analg 2011;38:415-422.

24. Bennett RC, Salla KM, Raekallio MR, et al. Effects of MK-467 on the antinociceptive and sedative actions and pharmacokinetics of medetomidine in dogs. $J$ Vet Pharmacol Ther 2016;39:336-343.

25. Honkavaara J, Restitutti F, Raekallio $M$, et al. Influence of MK-467, a peripherally acting alpha 2-adrenoceptor antagonist on the disposition of intravenous dexmedetomidine in dogs. Drug Metab Dispos 2012;40:445-449.

26. Ulger F, Bozkurt A, Bilge $S$, et al. The antinociceptive effects of intravenous dexmedetomidine in colorectal distensioninduced visceral pain in rats: the role of opioid receptors. Anesth Analg 2009;109:616-622.

27. Hamlin RL, Bednarski LS, Schuler CJ, et al. Method of objective assessment of analgesia in the dog. J Vet Pharmacol Ther 1988;11:215-220.

28. Santos-Nogueira E, Redondo Castro E, Mancuso R, et al. Randall-Selitto test: a new approach for the detection of neuropathic pain after spinal cord injury. $J$ Neurotrauma 2012;29:898-904

29. Houghton KJ, Rech RH, Sawyer DC, et al. Dose-response of intravenous butorphanol to increase visceral nociceptive threshold in dogs. Proc Soc Exp Biol Med 1991;197:290-296.

30. Sawyer DC, Rech RH, Durham RA, et al. Dose response to butorphanol administered subcutaneously to increase visceral nociceptive threshold in dogs. Am J Vet Res 1991;52:18261830.

31. European Medicines Agency. Guideline on bioanalytical method validation EMEA/CHMP/EWP/192217/2009. Available at: www.ema.europa.eu/en/documents/scientificguideline/guideline-bioanalytical-method-validation_en.pdf. Accessed Jun 17, 2019.

32. FDA. Guidance document. CVM GFI \#35 Bioequivalence Guidance. November 2006. Available at: www.fda. gov/downloads/AnimalVeterinary/GuidanceCompliance Enforcement/GuidanceforIndustry/UCM052363.pdf. Accessed Jul 13, 2019.

33. Sabbe MB, Penning JB, Ozaki GT, et al. Spinal and systemic action of the alpha 2 receptor agonist dexmedetomidine in dogs. Anesthesiology 1994;80:1057-1072.

34. van Oostrom H, Doornenbal A, Schot A, et al. Neurophysiological assessment of the sedative and analgesic effects of a constant rate infusion of dexmedetomidine in the dog. Vet J 2011;190:338-344.

35. Ness TJ, Gebhart GF. Colorectal distension as a noxious visceral stimulus: physiologic and pharmacologic characterization of pseudaffective reflexes in the rat. Brain Res 1988;450:153-169.

36. Ness TJ, Metcalf AM, Gebhart GF. A psychophysiological study in humans using phasic colonic distension as a noxious visceral stimulus. Pain 1990;43:377-386.

37. Ness TJ, Gebhart GF. Visceral pain: a review of experimental studies. Pain 1990;41:167-234.

38. Sanchez LC, Merritt AM. Colorectal distention in the horse: visceral sensitivity, rectal compliance and effect of i.v. xylazine or intrarectal lidocaine. Equine Vet J 2005;37:70-74.

39. Goligher JC, Hughes ES. Sensibility of the rectum and colon. Its role in the mechanism of anal continence. Lancet $1951 ; 1: 543-547$

40. Hector RC, Rezende ML, Mama KR, et al. Effects of constant 
rate infusions of dexmedetomidine or MK- 467 on the minimum alveolar concentration of sevoflurane in dogs. Vet Anaesth Analg 2017;44:755-765.

41. Antognini JF, Schwartz K. Exaggerated anesthetic requirements in the preferentially anesthetized brain. Anesthesiology 1993;79:1244-1249.

42. Rampil IJ, Mason P, Singh H. Anesthetic potency (MAC) is in dependent of forebrain structures in the rat. Anesthesiology 1993;78:707-712.

43. Antognini JF, Carstens E, Atherley R. Does the immobilizing effect of thiopental in brain exceed that of halothane? Anesthesiology 2002;96:980-986.

44. Pypendop BH, Ilkiw JE. Relationship between plasma dexmedetomidine concentration and sedation score and thermal threshold in cats. Am J Vet Res 2014;75:446-452.

45. Ansah OB, Raekallio M, Vainio O. Correlation between serum concentrations following continuous intravenous infusion of dexmedetomidine or medetomidine in cats and their sedative and analgesic effects. $J$ Vet Pharmacol Ther 2000;23:1-8.

46. Rolfe NG, Kerr CL, McDonell WN. Cardiopulmonary and sedative effects of the peripheral $\alpha_{2}$-adrenoceptor antagonist MK 0467 administered intravenously or intramuscularly concurrently with medetomidine in dogs. Am J Vet Res 2012;73:587-594

47. Iirola T, Vilo S, Aantaa R, et al. Dexmedetomidine inhibits gastric emptying and oro-caecal transit in healthy volunteers. Br J Anaesth 2011;106:522-527.

48. Vainionpää MH, Raekallio MR, Pakkanen SA, et al. Plasma drug concentrations and clinical effects of a peripheral alpha-2-adrenoceptor antagonist, MK-467, in horses sedated with detomidine. Vet Anaesth Analg 2013;40:257-264.

49. de Vries A, Pakkanen SAE, Raekallio MR, et al. Clinical effects and pharmacokinetic variables of romifidine and the peripheral $\alpha_{2}$-adrenoceptor antagonist MK-467 in horses. Vet Anaesth Analg 2016;43:599-610. 